Original Article

\title{
Changes in corticospinal excitability with short-du- ration high-frequency electrical muscle stimulation: a transcranial magnetic stimulation study
}

\author{
Kazuhiro Miyata, MS, $\mathrm{PT}^{1,2)^{*}}$, Shigeru Usuda, PhD, $\mathrm{PT}^{2)}$ \\ 1) Department of Rehabilitation Center, Hidaka Hospital: 886 Nakao-machi, Takasaki, Gunma \\ 370-0001, Japan \\ 2) Gunma University Graduate School of Health Sciences, Japan
}

\begin{abstract}
Purpose] Afferent input caused by electrical stimulation of a peripheral nerve or a muscle modulates corticospinal excitability. However, a long duration of stimulation is required to induce these effects. The purpose of this study was to investigate the effect of short-duration high-frequency electrical muscle stimulation (EMS) on corticospinal excitability through the measurement of motor evoked potentials (MEP) in young healthy subjects. [Subjects] Eleven healthy right-handed subjects participated in this study. [Methods] EMS was applied to the abductor pollicis brevis (APB) muscle at $100 \mathrm{~Hz}$ with a pulse width of $100 \mu \mathrm{s}$ for $120 \mathrm{~s}$. The intensity of stimulation was just below the motor threshold. Transcranial magnetic stimulation was applied over the motor cortex, and MEP were recorded from the APB before, and immediately, 10, and 20 min after EMS. [Results] In the APB muscle, the MEP amplitude significantly decreased after EMS, and this effect lasted for $20 \mathrm{~min}$. [Conclusion] The excitability of the corticospinal tract decreased after short-duration high-frequency EMS, and the effect lasted for 20 min. These results suggest that even short duration EMS can change the excitability of the corticospinal tract.

Key words: Electrical muscle stimulation, Transcranial magnetic stimulation, Corticospinal excitability
\end{abstract}

(This article was submitted Feb. 23, 2015, and was accepted Mar. 24, 2015)

\section{INTRODUCTION}

Stroke causes motor and sensory impairments that downgrade the quality of life. Impaired movement of the arm is common and is often the most troublesome problem experienced by stroke survivors. A recent systematic review ${ }^{1)}$ reported that the most promising intervention for restoring arm function was high-repetition doses of task-oriented training, such as constraint-induced movement therapy, mental practice, and robotics. However, a long duration is necessary for these treatments to be effective, and the adaptation range is limited. Sharma et al. $\left.{ }^{2}\right)$ suggested that cortical excitability should be modulated before motor practice to promote motor recovery or motor learning in stroke rehabilitation. There are two techniques to modulate cortical excitability: (1) noninvasive brain stimulation such as repetitive transcranial magnetic stimulation (TMS) or transcranial direct current stimulation, and (2) using somatosensory inputs from peripheral parts of the body elicited by vibration or electrical stimulation

Sensory input from a peripheral nerve changes the excit-

*Corresponding author. Kazuhiro Miyata (E-mail: ipukazuhiro@yahoo.co.jp)

(C2015 The Society of Physical Therapy Science. Published by IPEC Inc. This is an open-access article distributed under the terms of the Creative Commons Attribution Non-Commercial No Derivatives (by-ncnd) License $<$ http://creativecommons.org/licenses/by-nc-nd/3.0/> . ability of the cerebral cortex ${ }^{3-5)}$. The acquisition of accurate motor performance and motor learning of new motor skills requires somatosensory input. Electrical stimulation can generate somatosensory signals and is easy to use in the clinical setting, and some beneficial effects of electrical stimulation interventions have been reported. However, electrical stimulation parameters such as stimulation intensity, frequency, and duration were not consistent across studies, making it difficult for a systematic review ${ }^{6}$ to summarize the results.

Electrical stimulation can increase the activity of efferent motor fibers and/or increase the activity of afferent sensory fibers. Peripheral sensory nerve stimulation (PNS) is an electrical stimulation technique that increases the activity of afferent sensory fibers. PNS increases the excitability of the corticospinal pathway to the hand in healthy subjects, as evaluated by TMS delivered over the hand area of the motor cortex ${ }^{4,5,7,8)}$. PNS has been proposed as a supplemental therapy to facilitate the motor functions of stroke patients, such as pinching ${ }^{9)}$, swallowing ${ }^{10)}$, and performing hand $\operatorname{tasks}^{11,12)}$

In many studies, it has been reported that long-duration stimulation is necessary to change the excitability of the corticospinal tract. The shortest reported effective stimulation duration was the $45 \mathrm{~min}$ reported by Macky et al. ${ }^{7)}$. Although PNS is a potentially useful therapy, the PNS protocols that have been described are not practical for inpatient rehabilitation, as task-specific training is conducted after $2 \mathrm{~h}$ of PNS.

Uehara et al. $\left.{ }^{13}, 14\right)$ reported that high-frequency and shortduration (90 s) electrical muscle stimulation (EMS) applied 
to the muscles of the human hand reduced excessive motor activity, i.e., muscle hypertonia, by modulating the neural activity of the sensorimotor system. They evaluated the potential benefits of high-frequency EMS for hand motor skills, including whether or not the stimulation reduced non-essential motor activity potentially existing in the acquired motor skill, so as to upgrade the stabilized motor performance.

In a previous behavioral study ${ }^{15}$, we compared the effect of EMS for $120 \mathrm{~s}$ and PNS for $1 \mathrm{~h}$ before performance of a motor skill and found that although both improved motor performance, the improvement was greater after EMS than after PNS. We hypothesized that short-duration high-frequency EMS before motor practice would change the excitability of the corticospinal tract, just as has been reported for PNS. Therefore, the purpose of this study was to investigate the effect of short-duration high-frequency EMS on corticospinal excitability through the measurement of motor evoked potentials (MEP) in young healthy subjects.

\section{SUBJECTS AND METHODS}

Eleven healthy subjects participated in this study (two females, nine males; age range 23-29 years). All subjects were right-handed as measured by the Edinburgh Handedness Inventory ${ }^{16)}$. All subjects received an explanation of the experimental aims and procedures and their provided written informed consent. The Epidemiologic Research Ethics Committee of Gunma University Faculty of Medicine approved this protocol (No. 24-50).

The subjects were seated comfortably in a reclining chair and placed their right hand on a table. They were instructed to completely relax their hand and arm muscles throughout the experimental session. Surface electromyograms were recorded from the right abductor pollicis brevis (APB) muscle and first dorsal interosseous (FDI) muscle using disposable silver-silver chloride surface electrodes (5-mm diameter). The electromyogram signals were amplified, filtered with a bandwidth of $20-3,000 \mathrm{~Hz}$, and sampled at $10 \mathrm{kHz}$ using an A/D converter (Neuropack, Nihon Kohden, Tokyo, Japan).

EMS was delivered using a portable constant-current stimulator (TORIO 300, ITO P\&R, Tokyo, Japan) via a pair of electrical patches attached to the skin surface on the palm of the right hand over the APB. The anode patch was distally located (DC mode). EMS was performed for $120 \mathrm{~s}$ at $100 \mathrm{~Hz}$ with a pulse width of $100 \mu$ s. We used short-duration highfrequency EMS because this type of stimulation has been shown to reduce excessive motor activity and modulate sensorimotor neuronal state ${ }^{13,14)}$. The intensity of stimulation was set at just below the motor threshold in consideration of fatigue. This intensity of stimulation elicits no visible thumb movements and no muscle twitch, and was determined prior to the start of the experiment for each participant. The stimulation intensity ranged from 2 to $6 \mathrm{~mA}$.

TMS was delivered to the primary motor cortex (M1) in the left hemisphere using a figure-of-eight coil with an internal wing diameter of $70 \mathrm{~mm}$ (Magstim, Dyfed, UK). The coil was placed tangentially on the scalp with its handle pointing backward and rotated approximately $45^{\circ}$ away from the mid-sagittal line to induce a posterior-anterior current in the left M1. The optimal position for evoking motor evoked potentials (MEP) from the APB was determined and marked on a swimming cap worn by the subjects with a soft-tip pen to ensure reliable coil placement across trials. The resting motor threshold (rMT) was defined as the minimum stimulation intensity needed to evoke an MEP of more than $50 \mu \mathrm{V}$ in the APB in five out of 10 trials, and is expressed as the percentage of maximum stimulator output. The rMT and the amplitude of the MEP evoked by stimulation at 1.0, 1.2 and 1.4 times of the baseline rMT were determined at four time points: baseline, immediately after EMS (T0), 10 min after EMS (T1), and $20 \mathrm{~min}$ after EMS (T2). The three stimulation intensities were based on the baseline rMT that were administered randomly with an interstimulus interval of 5 to $7 \mathrm{~s}$, and 10 stimuli were delivered at each intensity. MEP amplitude was measured offline as the peak-to-peak amplitude of the electromyogram. MEP amplitude was measured for both the APB and the FDI. The MEP amplitude at T0, T1 and $\mathrm{T} 2$ were expressed as ratios of the mean MEP amplitude at baseline.

One-way repeated-measures analysis of variance (ANOVA) of time points (baseline, T0, T1, T2) was used to assess the effects of EMS on rMT and MEP amplitude. ANOVA was performed separately for each stimulation intensity (1.0, 1.2 , and 1.4 rMT) and each muscle (APB, FDI). Post-hoc pairwise comparisons were made using Dunnett's test. $\mathrm{P}$ values $<0.05$ were considered statistically significant. Statistical analyses were performed using IBM SPSS Statistics 20.

\section{RESULTS}

The rMT measured at baseline varied between $42 \%$ and $62 \%$ of maximum stimulator output. The mean rMT at baseline, T0, T1 and T2 were $49.5 \pm 5.2,49.9 \pm 4.8,49.8$ \pm 5.1 , and $49.6 \pm 5.3 \%( \pm \mathrm{SD})$, respectively. There was no significant main effect of time point on $\mathrm{rMT}(\mathrm{F}=0.793, \mathrm{p}>$ $0.05)$, indicating that rMT was not affected by EMS.

Typical MEP waveforms in the two muscles at baseline and at each time point after EMS are shown in Fig. 1. There was a main effect of time on the amplitude of the APB MEP elicited by stimulation of 1.2 rMT $(\mathrm{F}=4.981, \mathrm{p}<$ 0.01 ; Table 1). Post hoc comparisons revealed that the MEP amplitude at each post-EMS time point (T0, T1, T2) was significantly lower than the MEP amplitude at baseline ( $p$ $<0.05$ ). There was no main effect of time on the amplitude of the APB MEP elicited by the other stimulation intensities (1.0 rMT: $\mathrm{F}=0.257, \mathrm{p}=0.855 ; 1.4$ rMT: $\mathrm{F}=1.191, \mathrm{p}=$ 0.330 ), and no main effect of time on the amplitude of the FDI MEP for any stimulation intensity $(1.0 \mathrm{rMT}: \mathrm{F}=0.994$, $\mathrm{p}=0.409 ; 1.2 \mathrm{rMT}: \mathrm{F}=0.606, \mathrm{p}=0.616 ; 1.4 \mathrm{rMT}: \mathrm{F}=$ $2.633, \mathrm{p}=0.068)$.

\section{DISCUSSION}

The present study demonstrates that the excitability of the corticospinal tract was inhibited by short-duration high-frequency EMS applied to the APB, and that this effect continued for $20 \mathrm{~min}$. Furthermore, the effect was observed only in the APB, which was the target muscle of EMS, there being no change in the MEP elicited in the FDI. These results 
Table 1. Motor evoked potential ratios after electrical muscle stimulation

\begin{tabular}{lcccccc}
\hline & \multicolumn{3}{c}{ APB } & \multicolumn{3}{c}{ FDI } \\
\hline & $1.0 \mathrm{rMT}$ & $1.2 \mathrm{rMT}$ & $1.4 \mathrm{rMT}$ & $1.0 \mathrm{rMT}$ & $1.2 \mathrm{rMT}$ & $1.4 \mathrm{rMT}$ \\
\hline Baseline & $1.00 \pm 0.00$ & $1.00 \pm 0.00$ & $1.00 \pm 0.00$ & $1.00 \pm 0.00$ & $1.00 \pm 0.00$ & $1.00 \pm 0.00$ \\
T0 & $0.72 \pm 0.51$ & $0.69 \pm 0.25^{*}$ & $0.89 \pm 0.34$ & $1.47 \pm 1.36$ & $0.84 \pm 0.41$ & $0.87 \pm 0.26$ \\
T1 & $0.75 \pm 0.66$ & $0.61 \pm 0.26^{*}$ & $0.88 \pm 0.30$ & $1.36 \pm 1.29$ & $1.01 \pm 0.50$ & $0.90 \pm 0.26$ \\
T2 & $0.76 \pm 0.57$ & $0.64 \pm 0.35^{*}$ & $0.83 \pm 0.46$ & $1.36 \pm 0.99$ & $1.01 \pm 0.71$ & $0.78 \pm 0.21$ \\
\hline
\end{tabular}

Data are expressed relative to the amplitude at baseline and are the mean \pm SD

*: significantly different from the baseline measurement (pairwise comparison, $\mathrm{p}<0.05$ )

APB: abductor pollicis brevis, FDI: first dorsal interosseous, rMT: resting motor threshold.

T0: immediately after electrical muscle stimulation, T1: 10 min after electrical muscle stimulation, T2: 20 min after electrical muscle stimulation.

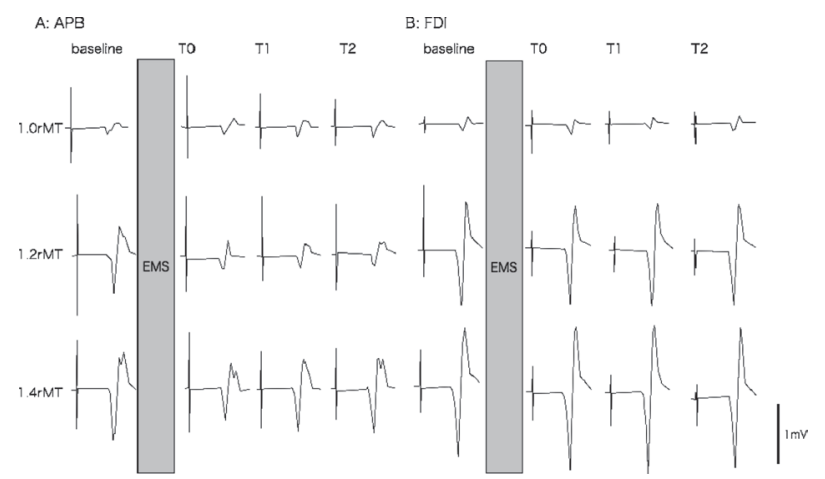

Fig 1. Representative motor evoked potentials waveforms in the abductor pollicis brevis (A) and first dorsal interosseous (B) muscles recorded from one subject before (baseline) and after (T0-T2) electrical muscle stimulation. The shaded area represents the period of electrical muscle stimulation. Transcranial magnetic stimulation was applied over the motor cortex at 1.0 (top), 1.2 (middle) and 1.4 (bottom) times resting motor threshold (rMT).

support the hypothesis that short-duration high-frequency EMS modulates the excitability of the corticospinal tract. The electrical stimulation parameters used in the present study changed the excitability of the corticospinal tract, even though electrical stimulation was only applied for a short duration, and the effect was sustained for 20 min, suggesting that it could be clinically useful.

The results of this study differ from many previous studies in which several tens of minutes or a few hours of electrical stimulation were required before effects were observed ${ }^{4-8)}$. Somatosensory inputs, such as electrical stimulation, influence the sensorimotor network within the cerebral cortex. In particular, it has been reported that sensory input from a muscle excites the cortex-striatum and cortex-cerebellum circuits $^{3)}$. Uehara et al. ${ }^{14)}$ reported that thenar muscle stimulation facilitated functional connectivity in corticosubcortical motor circuits, and improved performance of fine hand motor skills.

Electrical stimulation can either increase or decrease the excitability of the corticospinal tract. It is believed that there are two reasons why short-duration high-frequency EMS inhibits the excitability of the corticospinal tract. Frequency is cited as one of the reasons. Many previous studies using low-frequency (10-30 Hz) electrical stimulation have shown increased excitability of the corticospinal tract ${ }^{4-8)}$, whereas a few studies using high-frequency $(90-100 \mathrm{~Hz})$ electrical stimulation have shown decreased excitability ${ }^{17,18)}$. Mima et al. ${ }^{18)}$ reported that the APB MEP decreased after the thenar muscle was stimulated at $90 \mathrm{~Hz}$ for $30 \mathrm{~min}$. Thus, it is possible that stimulation at about $100 \mathrm{~Hz}$ inhibits excitability of the corticospinal tract. In the present study a stimulation of $100 \mathrm{~Hz}$ was used, and even though the duration of stimulation was short, inhibition of the corticospinal tract was observed, indicating that the stimulation parameters were appropriate. Sugawara et al. ${ }^{19)}$ investigated the influence of stimulation frequency by comparing the effects of stimulation at $1 \mathrm{~Hz}, 10 \mathrm{~Hz}, 100 \mathrm{~Hz}$, and 1,000 Hz. Although the excitability of the corticospinal tract tended to increase after stimulation at the higher frequencies, there were no statistically significant differences. Further studies of the relation between the frequency of electrical stimulation and its effects on the corticospinal tract are needed.

Muscle fatigue is a potential cause of decreased corticospinal tract excitability. It is possible that high-frequency stimulation of a muscle induces fatigue; however, in the present study the stimulation was below the motor threshold. When muscle fatigue is induced by voluntary contraction, the corticospinal tract is inhibited and intracortical inhibition is disinhibited ${ }^{20)}$. When muscle fatigue was induced by electrical stimulation above the motor threshold, as opposed to voluntary contraction, the excitability of the corticospinal tract initially increased before decreasing a few minutes later $^{21)}$. It is considered that the corticospinal tract is inhibited by muscle fatigue that is caused by voluntary contraction. However, there have been few studies of the effects of muscle fatigue caused by electrical stimulation. In this study, we only measured the MEP amplitude, which is thought to reflect the excitability of M1. However, the excitability of the spinal cord will also influence the amplitude of the MEP. Therefore, the MEP amplitude reflects the excitability of the whole corticospinal tract, indicating it will be necessary to measure M-waves to confirm the influence of muscle fatigue.

In this study MEP were recorded from the APB, which was the target muscle for EMS, and from the neighboring FDI muscle. The MEP amplitude in the APB decreased after EMS, and although there was a tendency of decrease, there was no statistically significant decrease in the MEP 
amplitude in the FDI. We suggest that this is related to the innervating nerve: the APB is innervated by the median nerve whereas the FDI is innervated by the ulnar nerve. A previous study ${ }^{4}$ reported that the MEP recorded from the abductor digiti minimi muscle increased after $2 \mathrm{~h}$ of PNS to the ulnar nerve, but the MEP recorded from the APB muscle did not change. This indicates the afferent somatosensory input from a muscle or a nerve is related to the innervating nerve.

A limitation of this study is that we used only MEP amplitude to quantify the effect of EMS. MEP amplitude reflects the excitability of the entire corticospinal tract, including the cortex and the spinal cord. To exclude the effect of spinal cord excitability on MEP amplitude it would be necessary to measure the amplitude of the $\mathrm{H}$ reflex. However, it has previously been reported that electrical stimulation of a peripheral nerve does not influence the excitability of the monosynaptic spinal cord reflex ${ }^{22)}$. Although there was inhibition of corticospinal tract excitability after EMS, we could not attribute this to a change in the excitability of M1. Therefore, it will be necessary to study the mechanism of the observed effects in more detail. When interpreting the results of this study, we could not exclude the influence of the placebo effect, nor discount the possibility that electrical stimulation increased attention on the stimulated part. Moreover, as plasticity in the injured brain differs from that in healthy subjects, the neurophysiological and behavioral effects of EMS need to be examined in patient populations.

The results of the study have clinical implications for the rehabilitation of stroke survivors receiving rehabilitation training utilizing unilateral afferent inputs, since EMS administered to an unaffected limb might modulate the undamaged M1. Depending on the impairment levels of motor and sensory functions, an intervention for the unaffected limb including EMS might be a more efficient method of stroke rehabilitation than an intervention for the affected limb. Furthermore, the advantage of EMS is that it more easily permits delivery of electrical stimulation in conjunction with task-oriented training and other exercises ${ }^{23-25)}$.

In conclusion, this study showed that a short-duration high-frequency EMS inhibited the excitability of the corticospinal tract to the APB. This finding provides a basis for the development of individual stimulation protocols in neurorehabilitation.

\section{REFERENCES}

1) Langhorne P, Coupar F, Pollock A: Motor recovery after stroke: a systematic review. Lancet Neurol, 2009, 8: 741-754. [Medline] [CrossRef]

2) Sharma N, Cohen LG: Recovery of motor function after stroke. Dev Psychobiol, 2012, 54: 254-262. [Medline] [CrossRef]

3) Naito E, Nakashima T, Kito T, et al.: Human limb-specific and non-limbspecific brain representations during kinesthetic illusory movements of the upper and lower extremities. Eur J Neurosci, 2007, 25: 3476-3487. [Medline] [CrossRef]

4) Kaelin-Lang A, Luft AR, Sawaki L, et al.: Modulation of human corticomotor excitability by somatosensory input. J Physiol, 2002, 540: 623-633. [Medline] [CrossRef]
5) Ridding MC, McKay DR, Thompson PD, et al.: Changes in corticomotor representations induced by prolonged peripheral nerve stimulation in humans. Clin Neurophysiol, 2001, 112: 1461-1469. [Medline] [CrossRef]

6) Chipchase LS, Schabrun SM, Hodges PW: Peripheral electrical stimulation to induce cortical plasticity: a systematic review of stimulus parameters. Clin Neurophysiol, 2011, 122: 456-463. [Medline] [CrossRef]

7) McKay D, Brooker R, Giacomin P, et al.: Time course of induction of increased human motor cortex excitability by nerve stimulation. Neuroreport, 2002, 13: 1271-1273. [Medline] [CrossRef]

8) Golaszewski SM, Bergmann J, Christova M, et al.: Increased motor cortical excitability after whole-hand electrical stimulation: a TMS study. Clin Neurophysiol, 2010, 121: 248-254. [Medline] [CrossRef]

9) Klaiput A, Kitisomprayoonkul W: Increased pinch strength in acute and subacute stroke patients after simultaneous median and ulnar sensory stimulation. Neurorehabil Neural Repair, 2009, 23: 351-356. [Medline] [CrossRef]

10) Fraser C, Power M, Hamdy S, et al.: Driving plasticity in human adult motor cortex is associated with improved motor function after brain injury. Neuron, 2002, 34: 831-840. [Medline] [CrossRef]

11) Ikuno K, Kawaguchi S, Kitabeppu S, et al.: Effects of peripheral sensory nerve stimulation plus task-oriented training on upper extremity function in patients with subacute stroke: a pilot randomized crossover trial. Clin Rehabil, 2012, 26: 999-1009. [Medline] [CrossRef]

12) Celnik P, Hummel F, Harris-Love M, et al.: Somatosensory stimulation enhances the effects of training functional hand tasks in patients with chronic stroke. Arch Phys Med Rehabil, 2007, 88: 1369-1376. [Medline] [CrossRef]

13) Uehara $\mathrm{S}$, Nambu I, Tomatsu $\mathrm{S}$, et al.: Improving human plateaued motor skill with somatic stimulation. PLoS ONE, 2011, 6: e25670. [Medline] [CrossRef]

14) Uehara S, Nambu I, Matsumura M, et al.: Prior somatic stimulation improves performance of acquires motor skill by facilitating functional connectivity in cortico-subcortical motor circuits. J Behav Brain Sci, 2012, 2: 343-356. [CrossRef]

15) Miyata K, Usuda S: Effect of two different types of electrical stimulation on upper-limb skill and pinch grip. Rigakuryouho Kagaku, 2014, 29: 315319 (in Japanese). [CrossRef]

16) Oldfield RC: The assessment and analysis of handedness: the Edinburgh inventory. Neuropsychologia, 1971, 9: 97-113. [Medline] [CrossRef]

17) Tinazzi $M$, Zarattini $S$, Valeriani $M$, et al.: Long-lasting modulation of human motor cortex following prolonged transcutaneous electrical nerve stimulation (TENS) of forearm muscles: evidence of reciprocal inhibition and facilitation. Exp Brain Res, 2005, 161: 457-464. [Medline] [CrossRef]

18) Mima T, Oga T, Rothwell J, et al.: Short-term high-frequency transcutaneous electrical nerve stimulation decreases human motor cortex excitability. Neurosci Lett, 2004, 355: 85-88. [Medline] [CrossRef]

19) Sugawara $K$, Tanabe $S$, Higashi $T$, et al.: Changes of excitability in $M 1$ induced by neuromuscular electrical stimulation differ between presence and absence of voluntary drive. Int J Rehabil Res, 2011, 34: 100-109. [Medline] [CrossRef]

20) Maruyama A, Matsunaga $K$, Tanaka N, et al.: Muscle fatigue decreases short-interval intracortical inhibition after exhaustive intermittent tasks. Clin Neurophysiol, 2006, 117: 864-870. [Medline] [CrossRef]

21) Pitcher JB, Miles TS: Alterations in corticospinal excitability with imposed vs. voluntary fatigue in human hand muscles. J Appl Physiol 1985, 2002, 92: 2131-2138. [Medline] [CrossRef]

22) Thompson AK, Doran B, Stein RB: Short-term effects of functional electrical stimulation on spinal excitatory and inhibitory reflexes in ankle extensor and flexor muscles. Exp Brain Res, 2006, 170: 216-226. [Medline] [CrossRef]

23) Kim H, Shim J: Investigation of the effects of mirror therapy on the upper extremity functions of stroke patients using the manual function test. $J$ Phys Ther Sci, 2015, 27: 227-229. [Medline] [CrossRef]

24) In TA, Jung KS, Lee SW, et al.: Virtual reality reflection therapy improves motor recovery and motor function in the upper extremities of people with chronic stroke. J Phys Ther Sci, 2012, 24: 339-343. [CrossRef]

25) Lee MH, Kim KD, Park SJ, et al.: The effects of bilateral activities of the upper extremities on stroke patient's daily living activities. J Phys Ther Sci, 2013, 25: 161-164. [CrossRef] 